\title{
Corrigendum: Anchoring the neural compass: coding of local spatial reference frames in human medial parietal lobe
}

\author{
Steven A Marchette, Lindsay K Vass, Jack Ryan \& Russell A Epstein \\ Nat. Neurosci. 17, 1598-1606 (2014); published online 5 October 2014; corrected after print 12 January 2015
}

In the version of this article initially published, three citations present in an earlier draft were omitted. To correct this, the following sentence and phrase have been inserted in the first paragraph of the Results section "Whole-brain analyses," before the third sentence and at the end of the fourth, respectively: "This locus is just posterior to retrosplenial cortex proper (Brodmann area 29/30), which has been previously implicated in the coding of spatially fixed objects ${ }^{59}$." “..., an area that has been previously implicated in the coding of directional information ${ }^{60,61}$." In the sentence before these inserts, the corresponding description "and just posterior to the retrosplenial cortex proper" has been deleted. The references are as follows:

59. Auger, S.D. \& Maguire, E.A. Assessing the mechanism of response in the retrosplenial cortex of good and poor navigators. Cortex 49, 2904-2913 (2013).

60. Schindler, A. \& Bartels, A. Parietal cortex codes for egocentric space beyond the field of view. Curr. Biol. 23, 177-182 (2013).

61. Spiers, H.J. \& Maguire, E.A. A navigational guidance system in the human brain. Hippocampus 17, 618-626 (2007).

The error has been corrected in the HTML and PDF versions of the article.

\section{Erratum: A region-specific neurogenesis mode requires migratory progenitors in the Drosophila visual system}

\author{
Holger Apitz \& Iris Salecker \\ Nat. Neurosci. 18, 46-55 (2015); published online 15 December 2014; corrected after print 23 December 2014
}

In the version of this article initially published, there were misworded sentences in the abstract and introduction and formatting errors in the genotypes in the Online Methods. The abstract referred to "neural stem cells and neuroblasts" where it should have read "neural stem cells (neuroblasts)." The fourth paragraph began, "The IPC produces a larval neuron population known as the distal cells, whose neurites in the adult extend into either the medulla and lobula or the medulla and lamina, lobula plate neurons, whose neurites connect the lobula plate with the medulla or lobula, and lobula neurons." The corrected sentence reads, "The IPC produces three populations: first, a larval neuron population known as the distal cells, whose neurites in the adult extend into either the medulla and lobula or the medulla and lamina; second, lobula plate neurons, whose neurites connect the lobula plate with the medulla or lobula; and third, lobula neurons." In the Online Methods, first paragraph, the following should have been superscripted: $x$ in $b r k x^{47}$ in all three instances, strII in $t k v s t r I I$ in item (5) of the second numbered list, 1 in "dac1 from F. Pignoni", IR KK100642 in UAS-fas3IR KK100642, and IR KK104691 in UAS-l'scIR KK104691. The following should not have been subscripted: $s c$ in UAS- $l^{\prime}{ }_{s c}$ IR TRiP.JF02399. The errors have been corrected in the HTML and PDF versions of the article.

\section{Corrigendum: Psychiatric genome-wide association study analyses implicate neuronal, immune and histone pathways}

The Network and Pathway Analysis Subgroup of the Psychiatric Genomics Consortium

Nat. Neurosci. 18, 199-209 (2015); published online 19 January 2015; corrected after print 19 January 2015

In the version of this article initially published, the affiliation numbers given for several authors were incorrect. Astrid M. Vicente was listed as associated with affiliations 234-236 instead of 233-235, Veronica J. Vieland as 237 instead of 236, Kenneth S. Kendler as 110, 253 and 254 instead of 110,252 and 253, and Zhaoming Zhao as 255 instead of 254 and 255. The errors have been corrected in the HTML and PDF versions of the article.

\section{Erratum: Visual recognition memory, manifested as long-term habituation, requires synaptic plasticity in $\mathrm{V} 1$}

\author{
Sam F Cooke, Robert W Komorowski, Eitan S Kaplan, Jeffrey P Gavornik \& Mark F Bear \\ Nat. Neurosci. 18, 262-271 (2015); published online 19 January 2015; corrected after print 9 February 2015
}

In the version of this article initially published, there were quotation marks around "encoded" in the first paragraph and around "encode" in the last paragraph of the main text; these have been deleted. The second sentence of the third paragraph read "habituated in a stimulus-selective manner in V1 as SRP developed"; "in V1" has been deleted. The Figure 7d legend began "Failure of SRP induction"; the correct text is "Selective failure of SRP expression." The sixth paragraph of the Discussion began "Behavioral manifestation of the vidget required V1"; the correct text is "Behavioral expression of the vidget requires V1." The eighth paragraph of the Discussion included "first, a 'response' pathway that directly mediates the vidget and does not undergo long-term modification, and second, a 'learning' pathway"; "first," and "second," have been deleted. The errors have been corrected in the HTML and PDF versions of the article. 